\title{
Regulation of erythrocyte lifespan: do reactive oxygen species set the clock?
}

\author{
Shilpa M. Hattangadi1,2 and Harvey F. Lodish'
}

${ }^{1}$ Whitehead Institute for Biomedical Research, Cambridge, Massachusetts, USA. ${ }^{2}$ Children's Hospital of Boston, Boston, Massachusetts, USA.

\begin{abstract}
The forkhead box $\mathrm{O}$ (Foxo) subfamily of transcription factors regulates expression of genes important for many cellular processes, ranging from initiation of cell cycle arrest and apoptosis to induction of DNA damage repair. Invertebrate Foxo orthologs such as DAF-16 also regulate longevity. Cellular responses inducing resistance to ROS are important for cellular survival and organism lifespan, but until recently, mammalian factors regulating resistance to oxidative stress have not been well characterized. Marinkovic and colleagues demonstrate in this issue of the JCI that Foxo3 is specifically required for induction of proteins that regulate the in vivo oxidative stress response in murine erythrocytes (see the related article beginning on page 2133). Their work offers the interesting hypothesis that in so doing, Foxo3 may regulate the lifespan of red blood cells, and underlies the importance of understanding the direct targets of this transcription factor and its regulation.
\end{abstract}

\section{Red blood cells:}

\section{the chief oxidative sink}

Red blood cell homeostasis is an excellent example of redox balance: erythroid progenitors accumulate hemoglobin during development, and erythrocytes continuously transport large amounts of oxygen over the course of their approximately 120 day lifespan. This results in a high level of oxidative stress (1). Fully mature red blood cells, lacking a nucleus, cannot produce new proteins in response to stress - they have to rely on proteins synthesized earlier in development to protect themselves from damage by ROS and thus ensure their own survival. Red blood cells have thus evolved to have an extensive array of antioxidants to counter this level of stress, including membrane oxidoreductases, cellular antioxidants such as catalase and superoxide dismutase (SOD), and enzymes that continuously produce reducing agents through the glutathione (GSH) system (2).

Defects in enzymes critical to the oxidative stress response have been implicated

Nonstandard abbreviations used: Foxo3, forkhead box O3; G6PD, glucose-6-phosphate dehydrogenase; GADD45, growth arrest and DNA damage-inducible 45; GSH, glutathione; MnSOD, mitochondrial SOD; NAC, $N$-acetyl-L-cysteine; SOD, superoxide dismutase.

Conflict of interest: H.F. Lodish receives research

funding from and is a consultant and expert witness for Amgen Inc. S.M. Hattangadi has no conflicts of interest to declare.

Citation for this article: J. Clin. Invest. 117:2075-2077 (2007). doi:10.1172/JCI32559. in human diseases ranging from mild chronic hemolysis to severe acute hemolysis (2). Because of a concomitant reduction in the normal red blood cell lifespan, these disease states are characterized by a compensatory increase in erythropoiesis, evident in patients as reticulocytosis. One relatively common genetic disorder is deficiency of glucose-6-phosphate dehydrogenase (G6PD), the enzyme that converts NADP to NADPH. NADPH is required for the maintenance of reduced GSH, and GSH in turn reduces peroxides, superoxides, and other ROS (2). G6PD is therefore required to protect the red blood cell from oxidative damage, and absence of this protection can result in severe hemolysis.

The deleterious effects of oxidative stress, such as damage to cellular proteins, DNA, and lipids, are well characterized. The dependence of the lifespan of the erythrocyte on an adequate antioxidant response has been previously demonstrated (3). However, the factors that regulate the oxidative stress response and the lifespan of erythrocytes are less clear.

\section{Foxo proteins: life or death?}

The subfamily of Foxo transcription factors includes four members, Foxo1, Foxo3, Foxo4, and Foxo6. All are known to control programs of gene expression regulating cellular stress resistance, apoptosis, and cell cycle arrest. Foxo proteins are crucial downstream targets of the PI3K-
AKT signal transduction pathway, which initiates cell survival and growth/metabolism pathways in response to activation of many growth factor receptors, such as the erythropoietin, insulin, and EGF receptors (4). When activated, the cytoplasmic serine/threonine kinase AKT phosphorylates its corresponding Foxo protein, resulting in sequestration of the transcription factor in the cytosol bound to a 14-3-3 protein (5) and thus inhibition of Foxodependent transcription. In the absence of growth factor signaling, Foxo proteins translocate into the nucleus. In some cells this results in induction of apoptosis by triggering expression of TNF-related apoptosis-inducing ligand (TRAIL), Fas ligand, and/or Bim proteins (6). Cell cycle arrest can be induced through activation of p27 (G1 arrest), growth arrest and DNA damage-inducible 45 (GADD45) expression (G2 arrest), or repression of cyclin D synthesis (G1 arrest) (7). DNA damage responses can be induced via induction of GADD45 and damage-specific DNA-binding protein 1 (DDB1) or stress resistance through upregulation of oxidative stress response proteins such as mitochondrial SOD (MnSOD) and catalase (8).

Foxo proteins also increase longevity in invertebrates: the Caenorhabditiselegans homo$\log$, DAF-16, activates genes promoting resistance to oxidative stress and repair of damaged cellular proteins. In worms, disruption of the PI3K pathway through mutation of the insulin receptor or PI3K results in continuous activation of DAF-16, prolonging longevity as much as 3 -fold (4). Importantly, this effect on lifespan is reversed when DAF-16 itself is mutated, indicating that DAF- 16 is the major protein in the insulin signaling pathway that regulates longevity (4). Similarly, overexpression of dFoxo - the single Foxo ortholog in Drosophila - increases longevity. Interestingly, mice lacking either the insulin receptor or the IGF1 receptor can live up to $30 \%$ longer than wild-type mice (4), suggesting that the role of Foxo factors in stress resistance and longevity is evolutionarily conserved, as are their target genes. 
A WT rbc

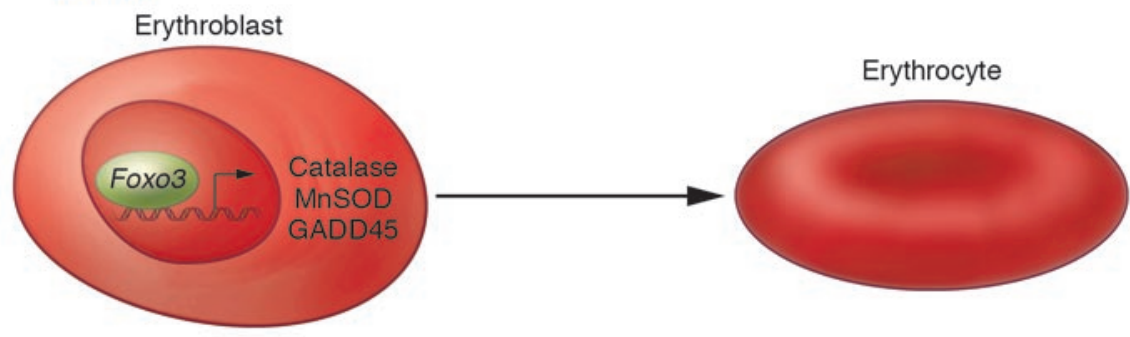

B Foxo3-null rbc

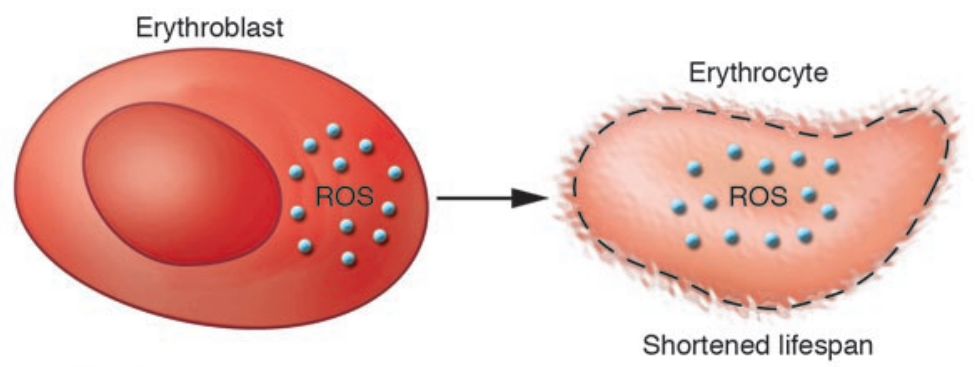

C Foxo3-null rbc + antioxidant

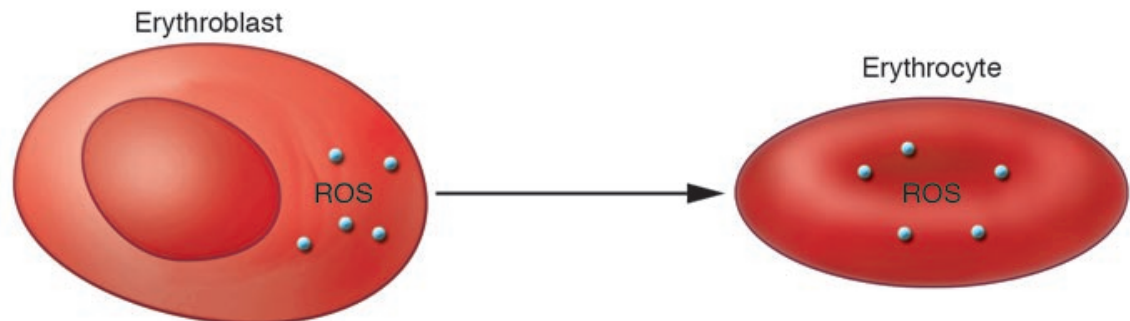

\section{Figure 1}

The effects of lack of Foxo3 on the erythrocyte lifespan. (A) Wild-type red blood cell development, in which Foxo3 induces the expression of antioxidants such as catalase, GADD45, and MnSOD in the erythroid progenitor. The terminally differentiated erythrocyte then develops normally and survives as expected (a mean of approximately 120 days). (B) As reported by Marinkovic et al. (10) in this issue of the $\mathrm{JCl}$, the lack of Foxo3 in erythroid progenitor cells of Foxo3-null mice results in an increase in ROS in the terminal red blood cell, which ultimately results in a reduction in red blood cell lifespan. These animals also had evidence of hemolytic anemia, including reticulocytosis and splenomegaly. This scenario is similar to that found in G6PD deficiency, in which lack of the enzyme that helps to convert NADP to NADPH results in hemolytic anemia and a shortened red blood cell lifespan. (C) Reversal of this detrimental effect on red blood cell lifespan by administration of exogenous antioxidant to the Foxo3-null animals, most likely due to the decrease in ROS.

\section{Foxo proteins also regulate the oxidative stress response}

Recently Tothova and colleagues (9) investigated the central role of the forkhead box proteins Foxo1, Foxo3, and Foxo 4 on cell cycle regulation and longevity of HSCs in mice. Using tissue-specific conditional knockouts, they found that mice lacking these Foxo proteins in the adult hematopoietic system had defects in both myeloid and lymphoid lineage expansion and also had fewer short- and long-term repopulating HSC populations, measured by the cell surface $\mathrm{Lin}^{-} \mathrm{Sca}{ }^{+}{ }^{+} \mathrm{Kit}^{+}$phenotype. There was a significant increase in ROS in the Foxo-null HSC population as compared with wild type, and this correlated with decreased expression of genes regulating the oxidative stress response. In addition, in vivo treatment with the ROS scavenger $N$-acetyl-L-cysteine (NAC) abrogated this phenotype in Foxo-null HSCs, indicating the major role of Foxo in response to ROS.

In this issue of the JCI, Marinkovic et al. (10) find, more specifically, that Foxo3 regulates expression of proteins required for in vivo oxidative stress responses in mature erythroid cells. Based on their initial observation that mice deficient in Foxo3 in hematopoietic cells die suddenly when exposed to oxidative stress induced by phenylhydrazine, the authors went on to demonstrate that loss of Foxo3 led to a reduction in erythrocyte lifespan as well as an enhanced mitotic arrest in intermediate $\left(\right.$ Ter $\left.119^{+} \mathrm{CD} 71^{+}\right)$erythroid progenitor cells (Figure 1). This resulted in a significant decrease in the rate of erythroid maturation. Foxo3-null erythrocytes also showed decreased expression of ROS-scavenging enzymes and evidence of oxidative damage. Interestingly, the authors also determined that these effects on erythrocyte lifespan are likely mediated by ROS, as the shortened lifespan of the Foxo3-null erythrocytes improved following treatment of the mice with the reducing agent NAC.

\section{Implications for erythrocyte lifespan: is the clock set?}

Marinkovic et al. (10) suggest that the mitotic arrest observed in Foxo3-null Ter $119^{+} \mathrm{CD} 71^{+}$erythrocyte precursors may be caused by p53-dependent G1-phase arrest induced as a response to stress through its

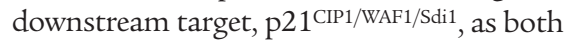
genes were upregulated in Foxo3-deficient erythroid precursors. There was also induction of the antioxidant p53 downstream targets GADD45 and sestrin 2 (SESN2). The authors speculate that the oxidative stress left unchecked by lack of Foxo3 likely turns on the $\mathrm{p} 53$ pathway in erythroid progenitors, resulting in induction of downstream targets that may mitigate oxidative stress and activate resistance to ROS-mediated damage.

Another interesting observation by Marinkovic et al. (10) was that the mitotic arrest and induction of $\mathrm{p} 21 \mathrm{CIP1} / \mathrm{WAF} 1 / \mathrm{Sdi1}$ in intermediate Foxo3-null erythroid progenitors was improved with NAC treatment. This raises the interesting possibility that ROS levels regulate Foxo3 or at least its function of initiating the oxidative stress response, creating a type of erythroid differentiation checkpoint. Hypothetically, when sufficient hemoglobin has been produced for the nascent terminal erythrocyte to carry out its oxygen-carrying tasks, the resulting oxidative stress from oxygen and 
heme moieties would somehow trigger Foxo3 translocation to the nucleus and induction of its targets in terminal differentiation. This as yet untested hypothesis emphasizes the importance of determining both the direct targets of Foxo3 and its own regulation in helping us understand how a red blood cell lacking a nucleus knows exactly when to die.

Address correspondence to: Harvey F. Lodish, Whitehead Institute for Biomedical Research, 9 Cambridge Center, Cam- bridge, Massachusetts, USA. Phone: (617) 258-5216; Fax: (617) 258-6768; E-mail: lodish@wi.mit.edu.

1. Fruehauf, J.P., and Meyskens, F.L., Jr. 2007. Reactive oxygen species: a breath of life or death? Clin. Cancer Res. 13:789-794.

2. McMullin, M.F. 1999. The molecular basis of disorders of red cell enzymes. J. Clin. Pathol. 52:241-244.

3. Tsantes, A.E., et al. 2006. Redox imbalance, macrocytosis, and RBC homeostasis. Antioxid. Redox Signal. 8:1205-1216.

4. Carter, M.E., and Brunet, A. 2007. FOXO transcription factors. Curr. Biol. 17:R113-R114.

5. Hermeking, H., and Benzinger, A. 2006. 14-3-3 proteins in cell cycle regulation. Semin. Cancer Biol.
16:183-192.

6. Burgering, B.M., and Kops, G.J. 2002. Cell cycle and death control: long live Forkheads. Trends Biochem. Sci. 27:352-360.

7. Furukawa-Hibi, Y., et al. 2005. FOXO transcription factors in cell-cycle regulation and the response to oxidative stress. Antioxid. Redox Signal. 7:752-760.

8. Tran, H., et al. 2002. DNA repair pathway stimulated by the forkhead transcription factor $\mathrm{FOXO} 3 \mathrm{a}$ through the Gadd45 protein. Science. 296:530-534.

9. Tothova, Z., et al. 2007. FoxOs are critical mediators of hematopoietic stem cell resistance to physiologic oxidative stress. Cell. 128:325-339.

10. Marinkovic, D., et al. 2007. Foxo3 is required for the regulation of oxidative stress in erythropoiesis. J. Clin. Invest. 117:2133-2144. doi:10.1172/ JCI31807.

\title{
Prime suspect: the TCF7L2 gene and type 2 diabetes risk
}

\author{
Andrew T. Hattersley \\ Institute of Biomedical and Clinical Sciences, Peninsula Medical School, Exeter, United Kingdom.
}

\begin{abstract}
Transcription factor-7-like 2 (TCF7L2) is the most important type 2 diabetes susceptibility gene identified to date, with common intronic variants strongly associated with diabetes in all major racial groups. This ubiquitous transcription factor in the Wnt signaling pathway was not previously known to be involved in glucose homeostasis, so defining the underlying mechanism(s) will provide new insights into diabetes. In this issue of the JCI, Lyssenko and colleagues report on their human and isolated islet studies and suggest that the risk allele increases $T C F 7 L 2$ expression in the pancreatic $\beta$ cell, reducing insulin secretion and hence predisposing the individual to diabetes (see the related article beginning on page 2155).
\end{abstract}

Over 170 million people in the world can blame their type 2 diabetes, at least in part, on their genes. It has been hoped for over 2 decades that identifying the "guilty" genes would help us to understand the fundamental pathophysiology of this common and important disorder. Now, at last, not only are common gene variants being reproducibly associated with type 2 diabetes, but work such as that of Lyssenko and colleagues, reported in this issue of JCI, is turning this genetic information into novel biological insights (1).

\section{Early genetic studies in type 2 diabetes}

Early attempts to identify the genes responsible for type 2 diabetes were slow and

Nonstandard abbreviations used: TCF $7 L 2$, transcription factor-7-like 2 gene.

Conflict of interest: The author has declared that no conflict of interest exists.

Citation for this article: J. Clin. Invest. 117:2077-2079 (2007). doi:10.1172/JCI33077. unsuccessful: faced with 30,000 suspects, geneticists were only able to examine less than $5 \%$ and, in most cases, the coverage of the gene and sample size were too small to detect modest effects. The choice of genes studied was primarily based on evidence that these genes played biologically important roles in glucose homeostasis. By the end of 2005, despite considerable research throughout the world, only 2 polymorphisms were considered guilty beyond a reasonable doubt of predisposing to type 2 diabetes: P12A in PPARG (2) and E23K in KCNJ11 (3). One advantage of using biological candidacy to choose genes for further study was that we already knew the critical science of the proteins encoded by these genes - the nuclear transcription factor PPAR $\gamma$ and the potassium inward-rectifying 6.2 subunit (Kir6.2) of the potassium ATP channel. Both genes were diabetes drug targets, and mutations in both could cause monogenic diabetes $(4,5)$. This meant that once the association with disease was established, understanding the associated pathophysiology was relatively straightforward. However, the very reasoning that led to the genes being chosen also meant there was not a lot of new scientific insights to be learned from identifying these two genes.

\section{TCF7L2: the most important type 2 diabetes gene}

At the start of 2006, transcription factor-7like 2 (TCF7L2) was revealed as an unexpected suspect for a type 2 diabetes gene by the DECODE group in Iceland (6). This gene had initially drawn attention during follow-up research on a small linkage signal on chromosome 10, but it turned out that, despite not explaining this linkage, multiple polymorphisms within the gene showed strong association with diabetes in multiple cohorts. The initial study was rapidly followed by widespread replication not only in white Europeans (7) but also in Indian and Japanese people (8-10), Mexican Americans (11), and West Africans (12) - representing the major racial groups with a high prevalence of type 2 diabetes. In all populations, TCF7L2 showed strong association, with the odds of developing type 2 diabetes being increased by $30 \%-50 \%$ for each allele inherited - approximately double the odds ratio seen with most other diabetes susceptibility polymorphisms.

The tracking of criminals and the tracking of genes have both been greatly helped by new technologies. Because of techno- 